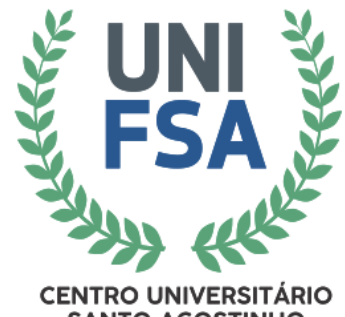

SANTO ACOSTINHO
CENTRO UNIVERSITÁRIO SANTO ACOSTINHO

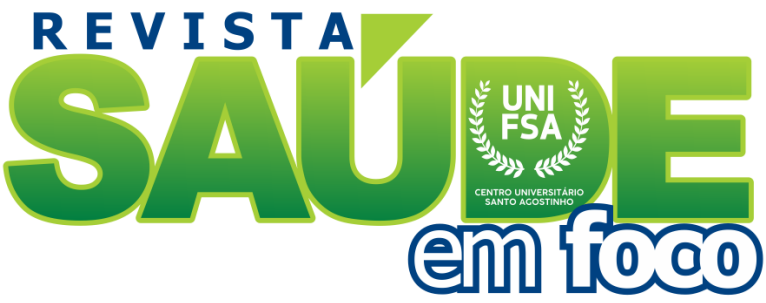

www4.fsanet.com.br/revista

Revista Saúde em Foco, Teresina, v. 8, n. 1, art. 4, p. 48-62, jan./abr. 2021

ISSN Eletrônico: 2358-7946

http://dx.doi.org/10.12819/rsf.2021.8.1.4

\title{
Uso Cosmético do Resveratrol no Controle do Envelhecimento Cutâneo Facial
}

\author{
Cosmetic Use of Resveratrol in the Control of Facial Cutaneous Aging
}

Janailde Pereira Mendes

Acadêmica do Curso de Estética e Cosmética do Centro Universitário Santo Agostinho

E-mail: janaildemendes12@gmail.com

Francismeire Mesquita de Souza

Acadêmica do Curso de Estética e Cosmética do Centro Universitário Santo Agostinho

E-mail: meirebel2015@gmail.com

Lyghia Maria Araújo Meirelles

Doutora em Desenvolvimento e Inovação Tecnológica de Medicamentos pela UFRN

Professora do Centro Universitário Santo Agostinho

E-mail: lyghiamaria@unifsa.com.br

Endereço: Janailde Pereira Mendes

Av. Valter Alencar 665 - São Pedro Teresina - PI - Cep: 64.019-625, Brasil.

Endereço: Francismeire Mesquita de Souza

Av. Valter Alencar 665 - São Pedro Teresina - PI - Cep: 64.019-625, Brasil.

Endereço: Lyghia Maria Araújo Meirelles

Av. Valter Alencar 665 - São Pedro Teresina - PI - Cep: 64.019-625, Brasil.
Editor-Chefe: Dr. Tonny Kerley de Alencar Rodrigues

Artigo recebido em 01/05/2021. Última versão recebida em 12/08/2021. Aprovado em 13/08/2021.

Avaliado pelo sistema Triple Review: a) Desk Review pelo Editor-Chefe; e b) Double Blind Review (avaliação cega por dois avaliadores da área).

Revisão: Gramatical, Normativa e de Formatação 


\title{
RESUMO
}

O envelhecimento tem sido um dos temas mais discutidos na atualidade, e diversas teorias tentam explicar esse processo. Porém, a teoria que envolve os radicais livres e as espécies reativas de oxigênio é uma das mais abordadas. O resveratrol (RSV), um importante polifenol presente principalmente na uva e seus derivados, tem sido amplamente pesquisado devido aos seus vários benefícios à saúde, incluindo as suas propriedades antioxidantes. Objetivo: Portanto, este trabalho pretendeu descrever a ação antioxidante do RSV, comprovando a sua aplicação cosmética no controle do envelhecimento cutâneo facial intrínseco e extrínseco. Metodologia: Trata-se de uma revisão integrativa da literatura, cujas buscas foram realizadas nas bases de dados da LILACS, SciELO e PubMed, utilizando os seguintes descritores: "Resveratrol", "Antioxidante", "Envelhecimento da Pele", "Cosmético" e "Estética". Foram incluídos artigos disponíveis na íntegra, publicados entre os anos 2016 e 2020, nos idiomas português ou inglês. Foram excluídos os artigos que não apresentaram compatibilidade com o tema, conforme triagem baseada na leitura do resumo, além de trabalhos de conclusão de curso, dissertações e teses. Resultados: Constatou-se que dentre os benefícios do RSV podem ser citados: a prevenção aos danos causados pelos radicais livres e radiação UV, o auxílio no clareamento da pele, uma ação antiacne, o aumento da hidratação da pele e o estímulo à produção de colágeno. Conclusão: O RSV demonstrou ter eficácia no controle do envelhecimento cutâneo facial intrínseco e extrínseco, podendo ser utilizado, também, como clareador da pele, porém, com resultados ainda limitados a poucos ensaios clínicos.

Palavras Chave: Antioxidante. Cosmético. Estética. Envelhecimento da Pele. Resveratrol.

\begin{abstract}
Aging has been one of the most discussed topics today, and several theories try to explain this process. However, the theory that involves free radicals and reactive oxygen species is one of the most discussed. Resveratrol (RSV), an important polyphenol present mainly in grapes and its derivatives, has been widely researched due to its various health benefits, including its antioxidant properties. Objective: Therefore, this work aimed to describe the antioxidant action of RSV, proving its cosmetic application in the control of intrinsic and extrinsic facial skin aging. Methodology: This is an integrative literature review, whose searches were carried out in the databases of LILACS, SciELO and PubMed, using the following descriptors: "Resveratrol", "Antioxidant", "Skin aging", "Cosmetic" and "Aesthetics". Articles available in full, published between the years 2016 and 2020, in Portuguese or English were included. Articles that did not show compatibility with the topic were excluded, according to screening based on reading the abstract, in addition to course completion papers, dissertations and doctoral thesis. Results: It was found that among the benefits of the RSV can be mentioned the prevention of damage caused by free radicals and UV radiation, the aid in whitening the skin, an anti-acne action, the increase in skin hydration and the stimulation of collagen production. Conclusion: RSV has proven to be effective in controlling intrinsic and extrinsic facial skin aging, and can also be used as a skin lightener, but with results still limited to a few clinical trials.
\end{abstract}

Keywords: Antioxidant. Cosmetic. Aesthetics. Skin aging. Resveratrol. 


\section{INTRODUÇÃO}

O corpo sofre mudanças fisiológicas ao longo do tempo, e um dos problemas mais importantes da atualidade é o envelhecimento, dado o aumento da expectativa de vida da população e a busca de formas de prolonga-la. Além de focar na saúde física e no bem-estar, há também a preocupação com o envelhecimento da pele, pois é crescente o número de pessoas que buscam intervenções para retardar ao máximo os sinais da idade avançada (PALADINI; LOPES; MACHADO, 2020).

Além disso, o estilo de vida da sociedade sofreu muitas mudanças no curso da vida moderna, principalmente urbana, com a exposição à poluição de modo mais acentuado, alimentação inadequada, ritmo de vida acelerado, estresse e sobrecarga de trabalho, entre outros fatores (SCHALKA, et. al., 2016). Dessa forma, o modo como o indivíduo envelhece tem causado preocupação, pois já não basta ter longevidade, é necessário viver com qualidade física e mental. Portanto, a busca por técnicas e substâncias que favoreçam o envelhecimento saudável tem estimulado novas pesquisas (PALADINI; LOPES; MACHADO, 2020).

O processo de envelhecimento está relacionado a fatores intrínsecos (fatores cronológicos) podendo ser decorrente da passagem do tempo, determinado principalmente por fatores genéticos, estado hormonal e reações metabólicas, como estresse oxidativo, além de fatores extrínsecos (fatores ambientais), como: radiação ultravioleta, tabagismo e obesidade. Esses interferentes levam a alterações no contorno e na elasticidade da pele, as quais manifestam-se sob a forma de rugas, sulcos, e dobras associados à flacidez. Tais sinais são causados principalmente pelo fotoenvelhecimento (UCKER; RIGO, 2019).

Dentre as substâncias cosméticas rejuvenescedoras, ou seja, utilizadas na prevenção e controle do envelhecimento cutâneo o RSV merece destaque, uma vez que é encontrado facilmente em diversas fontes naturais, como eucalipto, amendoim, amoras e principalmente na uva e seus derivados (PALADINI; LOPES; MACHADO, 2020).

Além disso, o RSV possui diversas atividades biológicas, incluindo funções cardioprotetoras e neuroprotetoras de combate ao envelhecimento e ao câncer, devido às suas propriedades antioxidantes. Logo, esse composto oferece proteção às células contra os danos oxidativos relacionados à ação dos radicais livres produzidos endogenamente e, principalmente, advindos de fontes extrínsecas, como as radiação ultravioleta responsáveis pelo fotoenvelhecimento. Por esse motivo, o RSV é cada vez mais utilizado em produtos com finalidade cosmética (RATZ-ŁYKO; ARCT, 2018). 
Dessa forma, o presente estudo tem como objetivo descrever a ação antioxidante do RSV comprovando a sua aplicação cosmética no controle ao envelhecimento cutâneo facial intrínseco e extrínseco.

\section{REFERENCIAL TEÓRICO}

\subsection{Processo de envelhecimento da pele}

A pele é o maior órgão do corpo humano, sendo uma camada de revestimento do organismo, fundamental para a manutenção da vida. Ademais, desempenha a função de isolar e proteger nosso organismo dos componentes do meio exterior, atuando como uma barreira de proteção (ANDRADE; CARVALHO, 2019).

Bernardo, et al. (2019) afirma que ela também possui a função de preservar a homeostase do organismo e a regulação de temperatura corpórea. A divisão da pele se dá em três camadas; Epiderme, Derme e a Hipoderme. A epiderme é a camada mais superficial e externa do corpo, a qual é formada por um revestimento de camadas de células sobrepostas. Por ser impermeável confere proteção contra microrganismos e agentes físicos e químicos do meio ambiente (DUARTE, 2012).

A camada intermediária da pele é chamada de derme, essa é a responsável pela nutrição de sua camada superior, a epiderme. Sua composição é formada de tecido conjuntivo vascularizado e nervoso, contendo folículos pilosos e glândulas sudoríparas e sebáceas. Seus principais componentes são o colágeno, substância que dá força à pele e é fundamental no processo cicatricial; e a elastina, proteína que fornece elasticidade e maleabilidade à pele (ANDRADE; CARVALHO, 2019).

Constituídas de colágeno e elastina, as fibras que compõem a derme funcionam como um motor do sistema tegumentar. Sendo o colágeno uma proteína fibrosa, esse é o componente mais abundante da matriz extracelular, fica responsável pela função de sustentar a estrutura da pele. Numa pele adulta existem cerca de $90 \%$ de colágeno tipo I e $10 \%$ de colágeno tipo III. Presume-se que haja uma redução da síntese e da quantidade total de colágeno em cerca de $1 \%$ ao ano, a partir da vida adulta (18 anos) por área de superfície da pele (GONZAGA, 2019).

A hipoderme ou tela subcutânea é formada por tecido conjuntivo frouxo com depósito de células adiposas, que têm como função a reserva energética, o isolamento térmico do organismo e a absorção de choques mecânicos. Essa camada de tecido adiposo embaixo da 
pele diminui com a idade, resultando em uma pele de espessura mais fina (CUNHA et al., 2019; GERSON et al., 2011; PANDOLFO, 2011).

O envelhecimento da pele é um fenômeno progressivo e degenerativo que ocasiona uma desorganização da matriz extracelular com a diminuição dos fibroblastos e fibrócitos contribuindo para a diminuição de suas funções (BERNARDO et al., 2019). Esse envelhecimento decorre de alterações morfológicas e funcionais que ocasionam a redução das células epiteliais, importantes para manutenção de equilíbrio corporal. Essas modificações ocorrem sob a influência de fatores extrínsecos e/ou intrínsecos (ADDOR, 2015).

A perda do colágeno no corpo tem início entre 18 e 29 anos, sendo cerca de $1 \%$ ao ano após 40 anos, e aproximadamente $75 \%$ depois dos 80 anos, em comparação aos adultos jovens. Além da idade, fatores como uma dieta deficiente em nutrientes, alcoolismo, tabagismo e algumas doenças, também contribuem para essa perda (FERREIRA et al., 2020).

Devido ao envelhecimento cutâneo a pele sofre algumas mutações que modificam a expressão de genes responsáveis pela síntese de enzimas e proteínas. Como consequência dessas modificações, há perda de capacidade de regular trocas aquosas, elasticidade e se intensificam as oxidações químicas e enzimáticas (JASKI et al., 2016).

\subsection{Radicais livres}

Os radicais livres são moléculas químicas instáveis, constituídas por um átomo ou a associação dos mesmos, o qual possui um elétron orbitando sua faixa externa. Essa distribuição eletrônica aduz grande instabilidade ao átomo que sempre busca capturar ou ceder elétrons das células que estão ao seu redor. Durante esse processo os RL deterioram a membrana plasmática e as organelas ao interagir com uma série de macromoléculas, levando em alguns casos à morte celular (LIU et al., 2015).

O processo de estresse oxidativo tem início devido à existência de uma desproporção entre compostos oxidantes e antioxidantes, em favor da produção excessiva de radicais livres ou em detrimento da velocidade de remoção desses. Dessa forma, o estresse oxidativo apresenta-se com um desequilíbrio que afeta o pleno funcionamento do organismo, contribuindo para o envelhecimento cutâneo e geral. No combate a esse desequilíbrio são empregadas substâncias antioxidantes (BARBOSA et al., 2010). 


\subsection{0 antioxidante resveratrol}

Existem substâncias que podem controlar esses efeitos danosos, elas são denominadas antioxidantes. Como o próprio nome sugere, atuam no combate direto aos RL, evitando o envelhecimento celular. Exemplo destes são os polifenóis, poderosos antioxidantes que possuem uma atuação anti-inflamatória e antioxidante, sendo capazes de sequestrar os RL produzidos pelas células. Os antioxidantes são um conjunto heterogêneo de substâncias formadas a partir de vitaminas, minerais, enzimas e outros compostos vegetais, que atuam num processo de combate e suavização dos efeitos maléficos causados pela presença excessiva de RL (GONZAGA, 2019).

Sua principal função é associada à capacidade de doar elétrons ao átomo desestabilizado com o objetivo de amenizar os processos oxidativos e, portanto, minimizar os danos moleculares nas células (GONZAGA, 2019).

Quanto à sua origem, estes são divididos em dois tipos: endógenos e exógenos. Os endógenos geralmente são constituídos por enzimas produzidas naturalmente pelo organismo, podendo ainda ser geradas por meio de absorção dietética. É sua função impedir o início do processo de oxidação por meio das proteínas antioxidantes, que têm a capacidade de ligar-se aos íons metálicos, com o papel de inibir a formação de RL (GONÇALVES, 2019).

Quanto aos exógenos, estes são compostos majoritariamente por vitaminas e outras substâncias adquiridas por meio de ingestão alimentar. Seu encargo consiste na interação com as moléculas de RL e sua destruição nesse processo. Essa proteção fornecida pelos antioxidantes é deveras abrangente, sendo um mecanismo de defesa que inibe a formação de RL (ADDOR, 2017).

Desta forma, os RL produzidos durante o metabolismo celular ou produzidos de forma exógena, impedem a degradação de moléculas como lipídios, aminoácidos e ácidos nucléicos. E, através de um mecanismo de reparo de lesões causadas pelos RL, ocorre a restauração de membranas celulares deterioradas (SILVA, 2018).

Nesse grupo vasto de antioxidante há o RSV, que protege o organismo dos efeitos nocivos dos RL, inclusive a pele, quando utilizado em forma estabilizada. Farmacologicamente, tem ação antiaging, clareadora, restaura o viço da pele, resultando em uma pele mais iluminada e hidratada. O resveratrol é um polifenol que pertence ao grupo das fitoalexina (FARRIS et al., 2013).

Essas são substâncias sintetizadas após a infecção por microrganismos para dificultar a proliferação, o crescimento e a propagação dos mesmos. Visto que sua formação abrange 
um composto antimicrobiano de baixa massa molecular, sintetizado pelas plantas em resposta à ação de agentes bióticos ou abióticos (MALHOTRA et al., 2015). Esse composto pode ser encontrado no rizoma da espécie Polygonum cuspidatum e na película de uvas (Vitis vinifera), além de estarem presentes em menor concentração nos arándanos, groselhas vermelhas e amendoins. Porém, a fonte mais comum do RSV é o vinho tinto, pois durante a produção da bebida há a liberação desse antioxidante em concentrações que variam entre $0,1-14,4 \mathrm{mg} / \mathrm{L}$, dependendo do tipo de uva utilizado, da região em que a mesma foi cultivada, dos fatores ambientais, da tecnologia de produção e do método de quantificação/detecção do RSV (ALVES, 2015).

Sobre o aspecto químico desse ativo natural, é importante citar que ele é considerado um polifenol. Os polifenóis foram apontados como os principais operadores para a manutenção de uma boa saúde cardiovascular nos povos itálicos (PERETTI et al., 2015). Cabe pontuar que os polifenóis são substâncias que contêm anéis fenólicos múltiplos. O RSV chega a ser até 10.000 vezes mais antioxidante do que a vitamina E. Além disso, inúmeros artigos relatam os benefícios do uso tópico do RSV, devido à sua atuação promovendo antienvelhecimento e dificultando a atividade da tirosinase (FRANCO et al., 2012).

Tirosinase é uma enzima que age na síntese da melanina, é uma enzima oxidativa, quando ativada por radiações ultravioletas ocorre o processo da melanogênese, esse processo ocorre dentro dos melanócitos. Quando esse processo é aumentado, pode ocorrer a hiperpigmentação e o envelhecimento extrínseco. O resveratrol apresenta diversas características para estender a expectativa de vida, aumentando a autoestima com um vigoroso antioxidante, atuando também como um anti-inflamatório cardioprotetor e demonstrando ação protetora contra a radiação solar. Ademais, tem efeito anti-inflamatório comprovado, mostrando-se eficaz como neuroprotetor e atuando na prevenção de doenças cardiovasculares. (ROSA et al., 2017).

\section{METODOLOGIA}

Trata-se de uma revisão da literatura do tipo integrativa, que visa descrever a ação antioxidante do RSV, comprovando a sua aplicação cosmética no controle do envelhecimento cutâneo facial intrínseco e extrínseco. Para a realização dessa revisão foram seguidas as seguintes etapas: elaboração da questão norteadora; estabelecimento dos critérios de busca, bem como dos critérios de inclusão e exclusão; análise dos estudos incluídos; e apresentação dos resultados. 
Nesse estudo foram incluídos artigos que analisam o mecanismo de ação do resveratrol no controle do envelhecimento da pele com destaque para a sua aplicação cosmética, publicados entre os anos 2016 e 2020, nos idiomas português ou inglês, cujo texto completo estava disponível. Foram excluídos os artigos que não apresentaram compatibilidade com o tema, conforme triagem baseada na leitura do resumo, além de trabalhos de conclusão de curso, dissertações e teses.

As buscas foram realizadas por meio das seguintes bases de dados da Literatura Latino-Americana e do Caribe em Ciências da Saúde (LILACS), Scientific Electronic Library Online (SciELO) e PubMed. Como estratégia de busca foram utilizados os descritores "Resveratrol", “Antioxidante", "Envelhecimento da Pele", "Cosmético", "Estética". selecionados de acordo com os Descritores em Ciências da Saúde (DeCS).

Após a seleção, os artigos foram organizados e analisados pelas pesquisadoras levando-se em consideração os seguintes dados: autores, ano de publicação, objetivo do estudo, principais resultados obtidos e conclusão. Após esse processo, foi elaborado um eixo temático para a discussão dos resultados. Para a apresentação dos resultados, foram criadas tabelas e figuras utilizando o Microsoft Office Excel (2010), descritas no decorrer do presente estudo, para melhor interpretação dos dados.

\section{RESULTADOS E DISCUSSÃO}

Após as buscas nas bases de dados, foram encontrados 11 estudos relacionados com o tema proposto. Os estudos mostraram uma eficácia na utilização do resveratrol para o rejuvenescimento. Dentre os 11 artigos pesquisados, boa parte deles apresenta pesquisas específicas sobre os efeitos desse produto para o rejuvenescimento, e todos esses estudos obtiveram bons resultados no que diz respeito à utilização do resveratrol para esse fim.

Ao analisar o potencial do RSV como agente de prevenção aos processos de envelhecimento celular, os autores concluíram que o RSV traz benefícios à saúde humana em relação ao envelhecimento cutâneo, uma vez que a substância ativa mecanismos de defesa celular que previnem os danos causados pelos radicais livres. Na época, os autores sugeriram a necessidade de realizarem-se mais estudos a fim de investigar tais efeitos da suplementação em longo prazo, a fim de estabelecer as doses corretas, podendo melhorar as alterações que levam ao envelhecimento cutâneo intrínseco e extrínseco (SILVA; CALEGARI, 2016). Desde então, alguns estudos mais recentes expõem respostas para as lacunas apontadas. 
De acordo com um levantamento realizado por Chool Boo (2019), o RSV pode clarear a pele humana e retardar o envelhecimento da pele por uma série de mecanismos: I) inibição direta da atividade catalítica da tirosinase humana; II) supressão da expressão gênica e maturação da tirosinase e outras enzimas melanogênicas; III) eliminação direta de ROS e/ou inibição de sua produção; IV) aumento da capacidade antioxidante por meio de mecanismos dependentes de um potente antioxidante; e V) um modulador de fator nuclear eritroide 2 relacionado ao fator 2 (Nrf2). Estudos clínicos apoiam a eficácia do RSV e seus análogos em humanos para o clareamento da pele e com fins antienvelhecimento. Em estudos com animais e em ensaios clínicos, $1 \%$ de resveratrol demonstrou reduzir a pigmentação induzida por UV quando é aplicado topicamente na pele.

Segundo Ratz-Lyko e Arct (2018), as formulações com RSV são capazes de estimular a proliferação de fibroblastos contribuindo para o aumento da concentração de colágeno III. Ademais, o RSV também possui afinidade com os receptores de proteínas de estrogênio (ERa e ER $\beta$ ), o que contribui para a estimulação da produção de colágeno tipo I e II. A reconhecida propriedade antioxidante do resveratrol pode ainda proteger as células contra danos oxidativos associados aos efeitos de radicais livres e à radiação UV na pele,

reduzindo a expressão dos fatores da proteína ativadora AP-1 e o fator nuclear NF$\mathrm{kB}$, retardando o processo de fotoenvelhecimento da pele. Nessa pesquisa, os resultados apontam para uma eficácia do RSV antienvelhecimento e anti-inflamatórias, bem como atividade de clareamento da pele e no tratamento antiacne.

Vale ressaltar, que os principais achados das pesquisas sobre o RSV referentes à sua aplicação cosmética se dão através de testes in vitro e escassos estudos in vivo, nos quais foram demonstradas as propriedades antioxidantes do RSV na fotoproteção e na redução da produção de melanina mediante a inibição não competitiva da tirosinase. O conhecimento sobre essas propriedades permite direcionar o foco para seu uso no rejuvenescimento, tanto sob a forma de cosméticos, como por meio da administração oral. Tais indícios têm despertado o interesse de empresas para o investimento de recursos na pesquisa e desenvolvimento de produtos à base do RSV (ACOSTA; THAUTHMAN, 2018).

Recentemente, o uso de alimentos funcionais surge como uma terapia alternativa para modular as alterações metabólicas relacionadas ao envelhecimento sistêmico. Por exemplo, o uso do RSV e da procaína pode controlar os efeitos oxidativos e inflamatórios na derme durante o envelhecimento crônico. Mediante o uso do RSV, detectou-se resistência aos danos causados pelos agentes estressantes como radiação UV e poluição do ar através da expressão de proteínas do choque térmico (HSPs), promovendo proteção, evitando interações 
incorretas entre proteínas, auxiliando na sua formação, síntese e dobramento (MOREIRA; SOARES; ROCHA, 2018).

Além disso, comprovou-se que quando um polifenol é utilizado em doses mais baixas, esse deve ser associado a um flavonoide, potencializando seu efeito dermatológico. De acordo com os resultados do estudo, ficou evidente a melhora da pele após a aplicação de pelling químico contendo RSV, bem como o aumento da espessura das camadas da pele. $\mathrm{O}$ RSV também possui eficácia na atenuação de manchas na pele, promovendo o clareamento da mesma, surgindo como uma terapia alternativa na amenização de manchas e rugas (MOREIRA; SOARES; ROCHA, 2018).

Seguindo a mesma linha de raciocínio, foi investigada a eficácia do arroz comestível normal (NR) e geneticamente modificado (RR), que produz o RSV, como um tratamento para o envelhecimento da pele. O RR mostrou-se particularmente eficiente em controlar o envelhecimento da pele induzido por raios UVB, mediado por metaloproteinases da matriz (MMP-1). Dessa forma, o estudo demonstrou o potencial do RR como um produto nutracêutico antienvelhecimento para a prevenção de complicações induzidas por UVB in vitro e ex vivo. Esse arroz enriquecido com RR supera as desvantagens do RSV e aumenta o seu potencial antienvelhecimento, uma vez que não exibe a toxicidade do antioxidante isolado, além de regular negativamente as vias subjacentes ao envelhecimento da pele induzidas por UVB (SUBEDI et. al., 2017).

A fim de melhorar a aplicação do RSV em alimentos e sistemas biológicos, Young e Shahidi (2017) modificaram-no estruturalmente, convertendo-o em éster a partir de 12 ácidos graxos diferentes. As novas moléculas derivadas de ácidos graxos de cadeia longa (C18:0 e C18:1) apresentaram maior atividade antioxidante frente ao radical DPPH, enquanto os ácidos graxos de cadeia curta (C3:0, C4:0 e C6:0) apresentaram maior atividade antioxidante no modelo que emprega o radical ABTS. Em relação aos derivados obtidos com ácidos graxos o resveratrol apresentou a maior atividade antioxidante. Os resultados das modificações estruturais evidenciaram que derivados lipofílicos de RSV podem ser benéficos antioxidantes para a saúde, os quais podem apresentar maior potencial de permeação cutânea.

Pelo fato de o RSV apresentar caráter predominantemente hidrofóbico, geralmente é incorporado em sistemas lipofílicos ou emulsionados. Dessa forma, pesquisadores propuseram um estudo controlado em um grupo de voluntários para avaliar o nível de hidratação da pele após o uso de um creme placebo versus um creme à base de RSV. O creme contendo RSV provou ser um cosmético eficaz na melhoria do funcionamento da barreira hidrolipídica da pele, aumentando o nível de hidratação da pele, evidenciando que o RSV 
penetra na epiderme complementando os lipídios de sua camada protetora. No decorrer de seis semanas de uso, as emulsões contendo RSV influenciaram positivamente o nível de hidratação da epiderme de modo significativo (KALWAT et. al., 2019).

Oliveira e colaboradores (2017) analisaram o potencial cosmético de um cristal líquido liotrópico contendo RSV para uso em voluntários humanos. A emulsão manteve-se estável durante os ensaios in vitro. A maioria dos voluntários não mostrou quaisquer alterações na pigmentação da pele ao longo do estudo. Mesmo assim, os voluntários relataram alto nível de satisfação com o produto, assim como recomendariam o cosmético para outras pessoas. $\mathrm{O}$ aumento da hidratação da pele e uma diminuição da oleosidade foram observados em mais da metade dos voluntários após sete dias de aplicação do produto. Dentre as melhorias mais significativas, pode ser citada a relação aos tamanhos dos poros na testa, que diminuíram, no entanto, as linhas de expressão não foram tão suavizadas. Porém, vale ressaltar que os resultados encontrados nesse estudo são conflitantes com a maioria dos relatos na literatura sobre o RSV para uso cosmético. Sugere-se que a formulação desenvolvida possa ter apresentado baixa taxa de cedência da molécula bioativa, limitando seus reconhecidos efeitos.

Além da aplicação cosmética tópica, alguns estudos propõem a administração do RSV pela via oral. Um estudo realizado recentemente verificou os benefícios do RSV no rejuvenescimento facial, constatando que após o uso oral, aproximadamente $70 \%$ do composto são absorvidos pelo organismo. Pequena parte da fração biodisponível permanece na circulação sistêmica na forma inalterada, sendo predominantes os metabólitos do RSV formados pelo processo de glicolisação e sulfatação. Para a aplicação tópica, por sua vez, é necessário investigar formas de potencializar a permeação cutânea do produto na pele, aumentando a eficácia do tratamento. Geralmente o RSV é adicionado a formulações de cremes ou óleos, que prolongam a sua liberação na pele. Porém, ao acrescentar o resveratrol em tais formulações, seu efeito é reduzido, como demonstrado anteriormente. Portanto, apesar do resveratrol ter se mostrado um ótimo aliado no controle do envelhecimento facial, fazemse importantes estudos que o veiculem em sistemas de liberação apropriados (SILVA; SILVA; SILVA, 2019).

Para tanto, a nanotecnologia tem sido explorada como plataforma tecnológica para muitos cosméticos já disponíveis no mercado. Por exemplo, o uso de dendrímeros se torna atrativo para os problemas de solubilidade apresentados pelo RSV. A principal vantagem da formulação de um creme baseado em dendrímero-RSV decorre da não utilização de solventes orgânicos nocivos para o corpo. Dessa forma, obteve-se uma nanoformulação aquosa de RSV, 
com maior permeação transdérmica, que amplia os benefícios de sua aplicação. O complexo dendrímero-resveratrol também aumenta a estabilidade do produto, auxiliando na manutenção das características físico-químicas de qualidade do produto por um longo período de tempo. Ademais, o complexo dendrimérico de RSV poderia ser incorporado em outras formulações de base aquosa, reduzindo custos e melhorando a sua biocompatibilidade (PENTEK et. al., 2017).

No entanto, apesar de todos os benefícios relatados, são necessários estudos futuros para avaliar criticamente a segurança do RSV, de seus análogos e seus metabólitos. O uso de antioxidantes em cosmetologia tem sido uma prática cada vez mais comum, tanto no aspecto oral quanto tópico, porém, muito mais pesquisas envolvendo RSV ainda são necessárias, não apenas para garantir a segurança desse composto em longo prazo, mas também para descobrir novas sinergias entre moléculas e, acima de tudo, novas formas de veiculação.

\section{CONSIDERAÇÕES FINAIS}

A exploração dos benefícios do RSV para fins cosméticos tem estimulado a realização de pesquisas com diversas abordagens. Nos últimos anos, por meio de muitos testes in vitro e uma pequena parcela de testes in vivo foram demonstradas as propriedades antioxidantes, fotoprotetoras e clareadoras do RSV. Diante do exposto, conclui-se que o RSV demonstrou ter eficácia no controle do envelhecimento cutâneo facial intrínseco e extrínseco, podendo ser utilizado também como clareador da pele, porém, com resultados ainda limitados. Boa parte dos estudos apontam positivamente para sua formulação e uso cosmético.

Porém, há uma lacuna persistente, poucos estudos clínicos em humanos visam esclarecer a segurança dos cosméticos à base de RSV. Essa demonstração se faz especialmente importante devido aos cosméticos à base de RSV serem classificados como grau II, devendo cumprir com requisitos junto à agência reguladora nacional.

\section{REFERÊNCIAS}

ACOSTA, C.B; TRAUTHMAN, S. C. Potencialidades cosméticas do resveratrol: uma visão geral. Trabalho de Conclusão de Curso. Universidade do Sul de Santa Catarina/ UNISUL, Tubarão, 2018.

ADDOR, F. A. S. Influência de um suplemento nutricional com peptídeos de colágeno nas propriedades da derme. Surgical and Cosmetic Dermatology, 7(2): 116-121. 2015. 
ADDOR, F. A. S. Antioxidants in dermatology. Anais Brasileiros de Dermatologia. [s.1.], v. 92, n. 3, p.356-362, 2017.

ALVES, A. C. L. O Resveratrol como Molécula Antienvelhecimento. Dissertação (Mestre em Ciências Farmacêuticas) - Universidade Lusófona de Humanidades e Tecnologias Faculdade de Ciências e Tecnologias da Saúde, Lisboa, 2015.

ANDRADE, R. C; CARVALHO, J. M. F. R. Rejuvenescimento Facial e as "Novas" Tecnologias. Revista Diálogos em Saúde, s. 1., v. 2, ed. 1, 2019.

BARBOSA, K. B. F. et al. Estresse Oxidativo: Conceito, Implicações e Fatores Modulatórios. Revista de Nutrição. São Paulo. Campinas, 23(4):629-643, 2010.

CUNHA, L. P. et al. Uso de Radiofrequência Como Tratamento Estético para Flacidez Tissular. XXIV Seminário Interinstitucional de Ensino, Pesquisa e Extensão - Ciência e Tecnologia para Transformação Social. 2019.

CHOOL BOO, Y. Human skin lightening efficacy of resveratrol and its analogs: from in vitro studies to cosmetic applications. Antioxidants, v. 8, p. 332. 2019.

DUARTE, A. B. A utilização da Radiofrequência como técnica de tratamento da flacidez corporal. Artigo Científico, 12 p. 2012.

FRANCO, D. et al. Inhibitory Effects of Resveratrol Analogs on Mushroom Tyrosinase Activity. Molecules, s.1., v.17, p. 11816-11825, 2012.

FERREIRA, A.S. Suplementação de colágeno e outras formas de tratamento no combate ao envelhecimento cutâneo: Revista Eletrônica Acervo Científico. s. 1., v. 12, ed. 4653, 10 2020 .

GERSON, J. et al. Fundamentos da Estética 3: Ciência da pele. 10. ed. São Paulo: Cengage Learning, 2011.

GONÇALVES, B. Hidrogel Formador De Filme Com Atividade Antioxidante Para Uso Tópico Contra O Envelhecimento Cutâneo: Uma Revisão Da Literatura. Trabalho de Conclusão de Curso - Universidade Federal de Santa Catarina, Centro de Ciências da Saúde. Santa Catarina. 2019. 48 f.

GONZAGA, S. A. S. Antioxidantes orais vs. Aplicação tópica na prevenção do envelhecimento cutâneo. Dissertação de Mestrado - Universidade Lusófona de Humanidades e Tecnologias - Faculdade de Ciências e Tecnologias da Saúde, Lisboa. 62p, 2019.

JASKI, M; LOTÉRIO, N; SILVA, D. A Ação De Alguns Antioxidantes no Processo do Envelhecimento Cutâneo. Santa Catarina, 2016.

KALWAT, J. I et al In vivo studies of resveratrol contained in cosmetic emulsions. Acta. ABP, Bioquimica Polomica. Vol. 66, N. 3, p. 371-374, 2019. 
LIU F.C; Tsai H.I; YU, H.P. Organ-Protective Effects of Red Wine Extract, Resveratrol, in Oxidative Stress-Mediated Reperfusion Injury. Oxidative Medicine and Cellular Longevity. 2015.

MALHOTRA, A; SUNDEEP, B. FAWZY, E. An Organ System Approach to Explore the Antioxidative, Anti-Inflammatory, and Cytoprotective Actions of Resveratrol. I Hindawi Publishing Corporation Oxidative Medicine and Cellular Longevity, v, 2015.

MOREIRA, L. M. C; SOARES, Y. S; ROCHA, B. P. Uma abordagem do resveratrol contra o envelhecimento cutâneo. VI Congresso internacional de envelhecimento humano. Universidade Estadual da Paraíba - UEPB, 2018.

OLIVEIRA, L. B. A et. al. Cosmetic Potential of a Liotropic Liquid Crystal Emulsion Containing Resveratrol. Cosmetics, 4, 54; 2017.

PALADINI; A.M; LOPES, T.D; MACHADO, K.E. Benefícios do resveratrol como ativo cosmético na prevenção do envelhecimento cutâneo. Infarma Ciências Farmacêuticas. v32. e4. p.319-328. 2020.

PANDOLFO, M. L. M. O processo de envelhecimento. Personalite. 2012. Disponível em: http://www.unifil.br/portal/arquivos/publicacoes/paginas/2012/8/485_769_publipg.pdf

PENTEK, T; NEWENHOUSE, E; O'BRIEN, B; CHAUHAN, A.S. Development of a Topical Resveratrol Formulation for Commercial Applications Using Dendrimer Nanotechnology Molecules, n. 22, p. 137. 2017.

PERETTI, S. C. et al. Resveratrol para cosméticos no clareamento da pele. Interfac EHS Saúde, Meio Ambiente e Sustentabilidade, São Paulo: Centro Universitário Senac, v. 10, n. ed. 1, junho 2015.

RATZ- $€$ YKO A, ARCT J. Resveratrol as an active ingredient for cosmetic and dermatological applications: a review. J. Cosmet. Laser. Ther, 21(2):84-90. 2018.

ROSA, M. de O. et al. O Efeito Protetor do Resveratrol na Doença de Alzheimer. Revista Brasileira Multidisciplinar - Revista Brasileira Multidisciplinar, ReBraM, v. 20, n.1, p 174 $-192,2017$.

SCHALKA, S. et al. Uma nova proposta para avaliação de cosmecêutico antioxidante no tratamento da pele afetada pelos efeitos da vida urbana. Surgical \& Cosmetic Dermatology, v.8, n.1, p. 46-54, 2016.

SUBEDI, L; LEE et al. Resveratrol-Enriched Rice Attenuates UVB-ROS-Induced Skin Aging via Downregulation of Inflammatory Cascades. Hindawi Oxidative Medicine and Cellular Longevity, Article, p. 15, 2017.

SILVA; I. K. M; CALEGARI, R.A. Análise das propriedades antienvelhecimento do resveratrol. $1^{\circ}$ Congresso nacional de iniciação cientifica. Centro Universitário Nossa Senhora do Patrocínio, Salto, SP. 2016. 
SILVA, A. P; SILVA, R. F; SILVA, D. P. Benefícios do resveratrol no rejuvenescimento facial. Revista Saúde em Foco, Edição no 11 - Ano: 2019.

ÜCKER, J. M; RIGO, M. P. M. Ação antioxidante do resveratrol no tratamento dermatológico: revisão de literatura. Revista Destaques Acadêmicos, Lajeado, v. 11, n. 3, 2019.

YOUNG OH, W; SHAHIDI, F. Lipophilization of Resveratrol and Effects on Antioxidant Activities. J. Agric. Food Chem. 65, 8617-8625. 2017.

YOON, H. S et al. Comparative Depigmentation Effects of Resveratrol and Its Two Methyl Analogues in $\alpha$-Melanocyte Stimulating Hormone-Triggered B16/F10 Murine Melanoma Cells Prev. Nutr. Food Sci. 21(2):155-159. 2016.

\section{Como Referenciar este Artigo, conforme ABNT:}

MENDES, J. P; SOUZA, F. M; MEIRELLES L. M. A. Uso Cosmético do Resveratrol no Controle do Envelhecimento Cutâneo Facial. Rev. Saúde em Foco, Teresina, v. 8, n. 1, art. 4, p. 48-62, jan./abr.2021.

\begin{tabular}{|l|c|c|c|}
\hline \multicolumn{1}{|c|}{ Contribuição dos Autores } & $\begin{array}{l}\text { J. P. } \\
\text { Mendes }\end{array}$ & $\begin{array}{l}\text { F. M. } \\
\text { Souza }\end{array}$ & $\begin{array}{l}\text { L. A. } \\
\text { Meirelles }\end{array}$ \\
\hline 1) concepção e planejamento. & $\mathrm{X}$ & $\mathrm{X}$ & $\mathrm{X}$ \\
\hline 2) análise e interpretação dos dados. & $\mathrm{X}$ & $\mathrm{X}$ & \\
\hline 3) elaboração do rascunho ou na revisão crítica do conteúdo. & $\mathrm{X}$ & $\mathrm{X}$ & \\
\hline 4) participação na aprovação da versão final do manuscrito. & & & $\mathrm{X}$ \\
\hline
\end{tabular}

